Marquette University

e-Publications@Marquette

Mathematics, Statistics and Computer Science

Mathematics, Statistics and Computer Science,

Faculty Research and Publications

Department of

$6-1-2000$

Some Applications of the Ultrapower Theorem to the Theory of Compacta

Paul Bankston

Marquette University, paul.bankston@marquette.edu

Accepted version. Applied Categorical Structures, Vol. 8, No. 1/2 (June 2000): 45-66. DOI. (C) 2000 Springer. Used with permission.

Shareable Link. Provided by the Springer Nature SharedIt content-sharing initiative. 


\title{
Some Applications of the Ultrapower Theorem to the Theory of Compacta
}

\author{
Paul Bankston \\ Department of Mathematics, Statistics and Computer Science \\ Marquette University \\ Milwaukee, WI 53201-1881 \\ paulb@mscs.mu.edu
}

Dedicated to Bernhard Banaschewski on the occasion of his 70th Birthday. A.M.S. Subject Classification (1991): 03C20, 54B35, 54C10, 54D05, 54D30, 54D80, 54F15, 54F45, 54F55.

Key Words and Phrases: ultraproduct, ultracoproduct, compactum, continuum, coelementary map, co-existential map.

\section{INTRODUCTION.}

In the ten years since the celebration of Professor Banaschewski's 60th birthday (and the writing of [4] in commemoration), there has been a fair amount of development in the theory of ultracoproducts of compacta (i.e., compact Hausdorff spaces). Papers $[3,5,7,6]$ have been written by this author; also there is the paper [13] by the late R. Gurevič. In addition to this, there has been a parallel development in the firstorder theory of Banach spaces/algebras begun by C. W. Henson in [16]. There are important links between Banach space theory and our work (mainly through Gel'fandNaĭmark duality), and we present here two applications of Banach techniques to the theory of compacta. (See 3.1 and 4.2 below.) Since Gel'fand-Naŭmark duality is a two-way street, much of the "dualized" model theory developed for compacta may be directly translated into the Banach model theory of commutative $B^{*}$-algebras. (See the results in $\S 5$ and $\S 6$ below.) It seems likely that the future will see much in the way of progress in these two streams of research, as topological issues stimulate the analytic and vice versa.

We begin with a quick review of the topological ultracoproduct construction; detailed accounts may be found in $[2,3,4,5,7,6,13]$.

We let $\mathbf{C H}$ denote the category of compacta and continuous maps. In model theory, it is well known that ultraproducts (and reduced products in general, but we restrict ourselves to maximal filters on the index set) may be described in the language of category theory; i.e., as direct limits of (cartesian) products, where the directed set is the ultrafilter with reverse inclusion, and the system of products consists of cartesian products taken over the various sets in the ultrafilter. (Bonding maps are just the obvious restriction maps.) When we transport this framework to $\mathbf{C H}$, the result is 
somewhat less than spectacular: If $\left\langle X_{i}: i \in I\right\rangle$ is a family of nonempty compacta and $\mathcal{D}$ is a nonprincipal ultrafilter on $I$, then the $\mathbf{C H}$-ultraproduct is degenerate (i.e., has only one point). What turns out to be vastly more fruitful, however, is the ultraproduct construction in the category-opposite of $\mathbf{C H}$; i.e., take an inverse limit of coproducts. The result is the topological ultracoproduct, and may be concretely (if opaquely) described as follows: Given $\left\langle X_{i}: i \in I\right\rangle$ and $\mathcal{D}$, let $Y$ be the disjoint union $\bigcup_{i \in I}\left(X_{i} \times\{i\}\right)$ (a locally compact space). With $q: Y \rightarrow I$ the natural projection onto the second coördinate (where $I$ has the discrete topology), we then have the Stone-Čech lifting $q^{\beta}: \beta(Y) \rightarrow \beta(I)$. Now the ultrafilter $\mathcal{D}$ may be naturally viewed as an element of $\beta(I)$, and it is not hard to show that the topological ultracoproduct $\sum_{\mathcal{D}} X_{i}$ is the pre-image $\left(q^{\beta}\right)^{-1}[\mathcal{D}]$. (The reader may be familiar with the Banach ultraproduct [10]. This construction is indeed the ultraproduct in the category of Banach spaces and nonexpansive linear maps, and may be telegraphically described using the recipe: take the usual ultraproduct, throw away the infinite elements, and mod out by the subspace of infinitesimals. Letting $C(X)$ denote the Banach space of continuous real-valued (or complex-valued) continuous functions with $X$ as domain, the Banach ultraproduct of $\left\langle C\left(X_{i}\right): i \in I\right\rangle$ via $\mathcal{D}$ is just $C\left(\sum_{\mathcal{D}} X_{i}\right)$.)

If $X_{i}=X$ for all $i \in I$, then we have the topological ultracopower $X I \backslash \mathcal{D}$, a subspace of $\beta(X \times I)$. In this case there is the Stone-Čech lifting $p^{\beta}$ of the natural first-coördinate map $p: X \times I \rightarrow X$. Its restriction to the ultracopower is a continuous surjection, called the codiagonal map, and is officially denoted $p_{X, \mathcal{D}}$ (with the occasional notation-shortening alias possible). This map is dual to the natural diagonal map from a relational structure to an ultrapower of that structure, and is not unlike the standard part map from nonstandard analysis.)

Many notions from classical first-order model theory, principally elementary equivalence, elementary embedding and existential embedding, may be phrased in terms of mapping conditions involving the ultraproduct construction. Because of the (KeislerShelah) ultrapower theorem (see, e.g., [9]), two relational structures are elementarily equivalent if and only if some ultrapower of one is isomorphic to some ultrapower of the other; a function from one relational structure to another is an elementary embedding if and only if there is an ultrapower isomorphism so that the obvious square mapping diagram commutes. A function $f: A \rightarrow B$ between relational structures is an existential embedding (i.e., making the image under $f$ a substructure of $B$ that is existentially closed in $B$ ) if and only if there are embeddings $g: A \rightarrow C, h: B \rightarrow C$ such that $g$ is elementary and equal to the composition $h f$. ( $C$ may be taken to be an ultrapower of $A$, with $g$ the natural diagonal.) (see also, e.g., $[2,5,11]$ ).

In $\mathbf{C H}$ one then constructs ultracoproducts, and talks of co-elementary equivalence, co-elementary maps and co-existential maps. Co-elementary equivalence is known $[2,5,13]$ to preserve important properties of topological spaces, such as being infinite, being a continuum (i.e., connected), being Boolean (i.e., totally disconnected), having (Lebesgue) covering dimension $n$, and being a decomposable continuum. If $f: X \rightarrow Y$ is a co-elementary map in $\mathbf{C H}$, then of course $X$ and $Y$ are co-elementarily equivalent $(X \equiv Y)$. Moreover, since $f$ is a continuous surjection (see [2]), additional information about $X$ is transferred to $Y$. For instance, continuous surjections in $\mathbf{C H}$ 
cannot raise weight (i.e., the smallest cardinality of a possible topological base, and for many reasons the right cardinal invariant to replace cardinality in the dualized model-theoretic setting), so metrizability (i.e., being of countable weight in the compact Hausdorff context) is preserved. Also local connectedness is preserved, since continuous surjections in $\mathbf{C H}$ are quotient maps. Neither of these properties is an invariant of co-elementary equivalence alone.

When attention is restricted to the full subcategory of Boolean spaces, the dualized model theory matches perfectly with the model theory of Boolean algebras because of Stone duality. In the larger category there is no such match [1, 24], however, and one is forced to look for other (less direct) model-theoretic aids. Fortunately there is a finitely axiomatizable AE Horn class of bounded distributive lattices, the so-called normal disjunctive lattices [6] (also called Wallman lattices in [5]), comprising precisely the (isomorphic copies of) lattice bases, those lattices that serve as bases for the closed sets of compacta. (To be more specific: The normal disjunctive lattices are precisely those bounded lattices $A$ such that there exists a compactum $X$ and a meet-dense sublattice $\mathcal{A}$ of the closed set lattice $F(X)$ of $X$ such that $A$ is isomorphic to $\mathcal{A}$.) We go from lattices to spaces, as in the case of Stone duality, via the maximal spectrum $S($ ), pioneered by H. Wallman [27]. $S(A)$ is the space of maximal proper filters of $A$; a typical basic closed set in $S(A)$ is the set $a^{\sharp}$ of elements of $S(A)$ containing a given element $a \in A . S(A)$ is generally compact with this topology. Normality, the condition that if $a$ and $b$ are disjoint $(a \sqcap b=\perp)$, then there are $a^{\prime}$, $b^{\prime}$ such that $a \sqcap a^{\prime}=b \sqcap b^{\prime}=\perp$ and $a^{\prime} \sqcup b^{\prime}=\top$, ensures that the maximal spectrum topology is Hausdorff. Disjunctivity, which says that for any two distinct lattice elements there is a nonbottom element that is below one of the first two elements and disjoint from the other, ensures that the map $a \mapsto a^{\sharp}$ takes $A$ isomorphically onto the canonical closed set base for $S(A)$. $S()$ is contravariantly functorial: If $f: A \rightarrow B$ is a homomorphism of normal disjunctive lattices and $M \in S(B)$, then $f^{S}(M)$ is the unique maximal filter extending the prime filter $f^{-1}[M]$. (For normal lattices, each prime filter is contained in a unique maximal one.) It is a fairly straightforward task to show, then, that $S($ ) converts ultraproducts to ultracoproducts, elementarily equivalent lattices to co-elementarily equivalent compacta, and elementary (resp., existential) embeddings to co-elementary (resp., co-existential) maps. Furthermore, if $f: A \rightarrow B$ is a separative embedding; i.e., an embedding such that if $b \sqcap c=\perp$ in $B$, then there exists $a \in A$ such that $f(a) \geq b$ and $f(a) \sqcap c=\perp$, then $f^{S}$ is a homeomorphism (see $[2,4,5,6,13]$ ). Because of this, there is much flexibility in how we may obtain $\sum_{\mathcal{D}} X_{i}$ : Simply choose a lattice base $\mathcal{A}_{i}$ for each $X_{i}$ and apply $S($ ) to the ultraproduct $\prod_{\mathcal{D}} \mathcal{A}_{i}$.

\section{The Topological Behavior of Co-existential Maps.}

Recall that a first-order formula in prenex form is an existential formula if all its quantifiers are existential. A function $f: A \rightarrow B$ between structures is an elementary (resp., existential) embedding if for every formula (resp. existential 
formula) $\varphi\left(x_{1}, \ldots, x_{n}\right)$ and every $n$-tuple $\left\langle a_{1}, \ldots, a_{n}\right\rangle$ from $A, A \models \varphi\left[a_{1}, \ldots, a_{n}\right]$ if and only if $B \models \varphi\left[f\left(a_{1}\right), \ldots, f\left(a_{n}\right)\right]$.

The ultrapower theorem states that a function $f: A \rightarrow B$ is an elementary embedding if and only if there is an isomorphism of ultrapowers $h: A^{I} / \mathcal{D} \rightarrow B^{J} / \mathcal{E}$ such that the obvious mapping square commutes; i.e., such that $d_{\mathcal{E}} f=h d_{\mathcal{D}}$, where $d_{\mathcal{D}}$ and $d_{\mathcal{E}}$ are the natural diagonal embeddings. There is also a characterization of existential embeddings along similar lines: $f: A \rightarrow B$ is an existential embedding if and only if there are embeddings $g: A \rightarrow C$ and $h: B \rightarrow C$ such that $g$ is elementary and $g=h f$. (By the ultrapower theorem, we may take $C$ to be an ultrapower $A^{I} / \mathcal{D}$ and $g=d_{\mathcal{D}}$ )

These characterizations have inspired the definition of the notions of co-elementary map and co-existential map in $\mathbf{C H}$ in terms of analogous mapping diagrams involving ultracopowers. Co-elementary (indeed, co-existential) maps are clearly continuous surjections. However, since specific situations involving co-elementary and co-existential maps are not guaranteed to correspond to analogous situations in elementary classes of relational structures, one may not take too much for granted. For example, there is no assurance a priori that the classes of co-elementary and coexistential maps are closed under composition or terminal factors (i.e., if both $f$ and $g f$ have a particular property, then $g$ has the property). As it happens, it is shown in [2] that closure under composition and terminal factors holds for co-elementary maps; also in [7] it is shown that there is an amalgamation property for co-elementary maps: If $f: Y \rightarrow X$ and $g: Z \rightarrow X$ are co-elementary, then there exist co-elementary maps $u: W \rightarrow Y$ and $v: W \rightarrow Z$ such that $f u=g v$. We do not know whether there is amalgamation for co-existential maps; however we can show closure under composition and terminal factors. We first prove a useful lemma.

2.1. Lemma. Let $\left\{f_{\delta}: X_{\delta} \rightarrow Y_{\delta}: \delta \in \Delta\right\}$ be a family of co-elementary and coexistential maps between compacta. Then there is a single ultrafilter witness to the fact. More precisely, there is an ultrafilter $\mathcal{D}$ on a set $I$ such that: $(i)$ if $f_{\delta}$ is co-elementary, then there is a homeomorphism $h_{\delta}: X_{\delta} I \backslash \mathcal{D} \rightarrow Y_{\delta} I \backslash \mathcal{D}$ such that $f_{\delta} p_{X_{\delta}, \mathcal{D}}=p_{Y_{\delta}, \mathcal{D}} h_{\delta}$; and $(i i)$ if $f_{\delta}$ is co-existential, then there is a continuous surjection $g_{\delta}: Y_{\delta} I \backslash \mathcal{D} \rightarrow X_{\delta}$ such that $f_{\delta} g_{\delta}=p_{Y_{\delta}, \mathcal{D}}$.

Proof. In S. Shelah's (GCH-free) proof of the ultrapower theorem (see [25]), he proves that if $\lambda$ is an infinite cardinal and $\mu=\min \left\{\kappa: \lambda^{\kappa}>\lambda\right\}$, then there is an ultrafilter $\mathcal{D}$ on $\lambda$ such that whenever $A$ and $B$ are elementarily equivalent relational structures of cardinality $<\mu$, then $A^{\lambda} / \mathcal{D}$ and $B^{\lambda} / \mathcal{D}$ are isomorphic. (We may easily extend this theorem to cover the situation involving elementary embeddings by adding constants to name domain elements. This way we get commuting square diagrams.)

Assume the maps $f_{\delta}$ are all co-elementary. (Co-existential maps are handled in a similar way.) Then we have a collection of homeomorphisms $k_{\delta}: X_{\delta} I_{\delta} \backslash \mathcal{D}_{\delta} \rightarrow Y_{\delta} J_{\delta} \backslash \mathcal{E}_{\delta}$ making the obvious mapping squares commute. Now, using Shelah's theorem, find 
an ultrafilter $\mathcal{D}$, on a very large index set $I$, so that the elementarity of the diagonal embeddings $d_{\delta}: F\left(X_{\delta}\right) \rightarrow F\left(X_{\delta}\right)^{I_{\delta}} / \mathcal{D}_{\delta}$ and $e_{\delta}: F\left(Y_{\delta}\right) \rightarrow F\left(Y_{\delta}\right)^{J_{\delta}} / \mathcal{E}_{\delta}$ (between normal disjunctive lattices), $\delta \in \Delta$, is simultaneously witnessed. When we apply the spectrum functor $S($ ) to these squares, we then have witnesses to the co-elementarity of the co-diagonal maps $p_{\delta}: X_{\delta} I_{\delta} \backslash \mathcal{D}_{\delta} \rightarrow X_{\delta}$ and $q_{\delta}: Y_{\delta} I_{\delta} \backslash \mathcal{E}_{\delta} \rightarrow Y_{\delta}$. (Recall that $S($ ) converts canonical ultrapower diagrams to canonical ultracopower diagrams, and that there is an iteration theorem for ultracopowers: "ultracopowers of ultracopowers are ultracopowers," see [2].) Now ( $) I \backslash \mathcal{D}$, as an operator on compacta, is functorial; it is covariant, and preserves (reflects as well) many interesting properties of continuous maps. In particular, as is straightforward to check, it preserves and reflects the properties of being surjective, of being one-one, of being co-elementary, and of being co-existential. Thus, when we apply ( $) \backslash \backslash \mathcal{D}$ to the homeomorphisms $k_{\delta}$, we have a new collection of homeomorphisms; hence we have a homeomorphism linkage between $X_{\delta} I \backslash \mathcal{D}$ and $Y_{\delta} I \backslash \mathcal{D}$, witnessing the co-elementarity of $f_{\delta} . \dashv$

2.2. Proposition. Let $f: X \rightarrow Y$ and $g: Y \rightarrow Z$ be functions between compacta. If $f$ and $g$ are co-existential maps, then so is $g f$; if $g f$ is a co-existential map and $f$ is a continuous surjection, then $g$ is a co-existential map.

Proof. Note that the second assertion above says more than just closure under terminal factors; we do not assume the co-existentiality of $f$. The proof of this is immediate from the definition, so we now consider the issue of closure under composition. Using 2.1, we have $p_{Y, \mathcal{D}}: Y I \backslash \mathcal{D} \rightarrow Y, u: Y I \backslash \mathcal{D} \rightarrow X, p_{Z, \mathcal{D}}: Z I \backslash \mathcal{D} \rightarrow Z$, and $v: Z I \backslash \mathcal{D} \rightarrow Y$, witnessing the co-existentiality of $f$ and $g$. Since $g$ is co-existential, so is the ultracopower map $g I \backslash \mathcal{D}$. Let $r: W \rightarrow Z I \backslash \mathcal{D}$ and $s: W \rightarrow Y I \backslash \mathcal{D}$ witness the fact. (So $r$ is co-elementary and $s$ is a continuous surjection.) Then, because compositions of co-elementary maps are co-elementary, we have a witness to the conclusion that $g f$ is a co-existential map. $\dashv$

Before proceeding, some notation is in order. If $\left\langle X_{i}: i \in I\right\rangle$ is a family of compacta and $\mathcal{D}$ is an ultrafilter on $I$, then, as noted before, $\sum_{\mathcal{D}} X_{i}$ may be viewed as the space of maximal filters of the normal disjunctive lattice $\prod_{\mathcal{D}} F\left(X_{i}\right)$. Let $A_{i} \subseteq X_{i}$ for each $i \in I$. Then we extend the "sharp" notation mentioned above by defining $\left(\prod_{\mathcal{D}} A_{i}\right)^{\sharp}:=\left\{m \in \sum_{\mathcal{D}} X_{i}: \prod_{\mathcal{D}} A_{i}\right.$ contains a member of $\left.m\right\} .\left(\prod_{\mathcal{D}} A_{i}\right)^{\sharp}$ is closed (resp., open) in the ultracopower if and only if $\left\{i \in I: A_{i}\right.$ is closed (resp., open) in $\left.X_{i}\right\} \in \mathcal{D}$. Of course, if each $A_{i}$ is closed in $X_{i}$, and therefore a compactum in its own right, there is a natural homeomorphism between $\sum_{\mathcal{D}} A_{i}$ and the subspace $\left(\prod_{\mathcal{D}} A_{i}\right)^{\sharp}$.

The following lemma tells us what happens to pre-images of open sets under codiagonal maps. We use an overline to indicate topological closure. 
2.3. Lemma. Let $U$ be an open subset of the compactum $X$. Then $p_{X, \mathcal{D}}^{-1}[U]=$ $\bigcup\left\{\left(\bar{V}^{I} / \mathcal{D}\right)^{\sharp}: \bar{V} \subseteq U, V\right.$ open in $\left.X\right\} \subseteq\left(U^{I} / \mathcal{D}\right)^{\sharp}$.

Proof. $p_{X, \mathcal{D}}(m)=x$ if and only if for each open neighborhood $W$ of $x, m \in\left(W^{I} / \mathcal{D}\right)^{\sharp}$. Suppose $m \in p_{X, \mathcal{D}}^{-1}[U]$, say $p_{X, \mathcal{D}}(m)=x$. Let $V$ be any open set with $x \in V \subseteq \bar{V} \subseteq U$. Then $m \in\left(V^{I} / \mathcal{D}\right)^{\sharp} \subseteq\left(\bar{V}^{I} / \mathcal{D}\right)^{\sharp}$.

For the reverse inclusion, suppose $x=p_{X, \mathcal{D}}(m) \notin U$. Pick $V$ open with $\bar{V} \subseteq U$, and let $W$ be an open neighborhood of $x$ such that $\bar{W} \cap \bar{V}=\emptyset$. Then $\bar{W}^{I} / \mathcal{D} \in m$. But $\bar{W}^{I} / \mathcal{D} \cap \bar{V}^{I} / \mathcal{D}=(\bar{W} \cap \bar{V})^{I} / \mathcal{D}=\emptyset$, so $\bar{V}^{I} / \mathcal{D} \notin m$; i.e., $m \notin\left(\bar{V}^{I} / \mathcal{D}\right)^{\sharp}$. $\dashv$

2.4. Theorem. Let $f: X \rightarrow Y$ be a co-existential map between compacta. Then there exists a $\cup$-semilattice homomorphism $f^{*}$ from the subcompacta of $Y$ to the subcompacta of $X$ such that for each subcompactum $K$ of $Y:(i) f\left[f^{*}(K)\right]=K$; (ii) $f^{-1}[U] \subseteq f^{*}(K)$ whenever $U$ is open and $U \subseteq K$; $($ iii $)$ the restriction $f \mid f^{*}(K)$ is a co-existential map from $f^{*}(K)$ to $K$; and $(i v) f^{*}(K) \in \mathbf{K}$ whenever $K \in \mathbf{K}$ and $\mathbf{K} \subseteq \mathbf{C H}$ is closed under ultracopowers and continuous images.

Proof. Let $g: Y I \backslash \mathcal{D} \rightarrow X$ witness the fact that $f$ is a co-existential map (i.e., $\left.f g=p_{\mathcal{D}}\right)$, and let $K$ be a subcompactum of $Y$. Then $\left(K^{I} / \mathcal{D}\right)^{\sharp}$ is a subcompactum of $Y I \backslash \mathcal{D}$, naturally homeomorphic to $K I \backslash \mathcal{D}$, and $p_{\mathcal{D}} \mid\left(K^{I} / \mathcal{D}\right)^{\sharp}$ may be viewed as the natural codiagonal from $K I \backslash \mathcal{D}$ to $K$. Thus $f^{*}(K):=g\left[\left(K^{I} / \mathcal{D}\right)^{\sharp}\right]$ is a subcompactum of $X$, and $f \mid f^{*}(K)$ is a co-existential map onto its image $K$. That condition $(i v)$ is true is now apparent.

Suppose $U$ is open in $Y$, with $U \subseteq K$. Then $f^{-1}[U]=g\left[p_{\mathcal{D}}^{-1}[U]\right]$. But by 2.3, $p_{\mathcal{D}}^{-1}[U] \subseteq\left(U^{I} / \mathcal{D}\right)^{\sharp} \subseteq\left(K^{I} / \mathcal{D}\right)^{\sharp}$. Thus $f^{-1}[U] \subseteq f^{*}(K)$.

It remains to show that the mapping $f^{*}$ is a $U$-semilattice homomorphism. If $K_{1}$ and $K_{2}$ are subcompacta of $Y$, then $f^{*}\left(K_{1} \cup K_{2}\right)=g\left[\left(\left(K_{1} \cup K_{2}\right)^{I} / \mathcal{D}\right)^{\sharp}\right]=$ $g\left[\left(K_{1}^{I} / \mathcal{D}\right)^{\sharp} \cup\left(K_{2}^{I} / \mathcal{D}\right)^{\sharp}\right]=f^{*}\left(K_{1}\right) \cup f^{*}\left(K_{2}\right)$. Clearly $f^{*}(\emptyset)=\emptyset$ and $f^{*}(Y)=X$. $\dashv$

A number of properties, not generally preserved by continuous surjections between compacta, are now easily seen to be preserved by co-existential maps.

2.5. Proposition. The following properties are preserved by co-existential maps: $(i)$ being infinite; (ii) being disconnected; (iii) being a Boolean space; ( $i v$ ) being an indecomposable continuum; and $(v)$ being a hereditarily indecomposable continuum.

Proof: Let $f, X, Y, g, \mathcal{D}$ and $K$ be as in the proof of 2.4. If $Y$ is finite (resp., connected), then so is $Y I \backslash \mathcal{D}$, and hence $X$. If $Y$ is not Boolean, then we may choose $K$ to be an infinite subcontinuum of $Y$. This forces $f^{*}(K)$ to be an infinite subcontinuum of $X$. 
Suppose $X$ is an indecomposable continuum (so there is no way to write $X$ as the union of two proper subcontinua). Then $Y$ is a continuum. Suppose $Y=K_{1} \cup$ $K_{2}$, where each $K_{n}$ is a proper subcontinuum. Then each $f^{*}\left(K_{n}\right)$ is also a proper subcontinuum of $X$, and $f^{*}\left(K_{1}\right) \cup f^{*}\left(K_{2}\right)=f^{*}\left(K_{1} \cup K_{2}\right)=f^{*}(Y)=f^{-1}[Y]=X$. This contradicts the assumption that $X$ is indecomposable.

Suppose $X$ is a hereditarily indecomposable continuum (so no subcontinuum of $X$ is decomposable). If $K$ is a decomposable subcontinuum of $Y$, then $f^{*}(K)$ is a decomposable subcontinuum of $X$, since $f \mid f^{*}(K)$ is a co-existential map onto $K$. (Use the result of the last paragraph.) $\dashv$

We can actually improve on 2.5(iii). Noting that being a Boolean space means being of covering dimension zero, it is tempting to conjecture that co-existential maps cannot raise dimension. This turns out to be the case, but it seems we need more than just 2.4 for a proof. For us, the handiest version of "being of dimension $\leq n$ " is due to E. Hemmingsen (see [12]): A normal space $Y$ is of covering dimension $\leq n, n<\omega,(\operatorname{dim}(Y) \leq n)$ if whenever $B_{1}, \ldots, B_{n+2}$ is a family of closed subsets of $Y$ and $B_{1} \cap \ldots \cap B_{n+2}=\emptyset$, then there is a family $F_{1}, \ldots, F_{n+2}$ of closed subsets of $Y$ such that $F_{m} \supseteq B_{m}$ for each $0 \leq m \leq n+2, F_{1} \cap \ldots \cap F_{n+2}=\emptyset$, and $F_{1} \cup \ldots \cup F_{n+2}=Y$.

2.6. Theorem. Suppose $f: X \rightarrow Y$ is a co-existential map between compacta, $n<\omega$. If $\operatorname{dim}(X) \leq n$, then $\operatorname{dim}(Y) \leq n$.

Proof. Let $B_{1}, \ldots, B_{n+2} \subseteq Y$ be given, as per the characterization given above. Let $g: Y I \backslash \mathcal{D} \rightarrow X$ witness the fact that $f$ is a co-existential map, and set $A_{m}:=f^{-1}\left[B_{m}\right]$, $1 \leq m \leq n+2$. Each $A_{m}$ is closed in $X$, and $A_{1} \cap \ldots \cap A_{n+2}=\emptyset$. Since $\operatorname{dim}(X) \leq n$, we find appropriate closed sets $E_{m} \supseteq A_{m}, 1 \leq m \leq n+2$, witnessing the fact. Pull these sets back to the ultrapower via $g$. We get a closed cover whose intersection is empty. Using compactness, we can obtain basic closed sets $\sum_{\mathcal{D}} F_{m, i} \supseteq g^{-1}\left[E_{m}\right] \supseteq B_{m} I \backslash \mathcal{D}$, $1 \leq m \leq n+2$, such that $\bigcap_{m=1}^{n+2} \sum_{\mathcal{D}} F_{m, i}=\emptyset$. By elementary ultraproduct considerations, we then have $\left\{i \in I: F_{m, i} \supseteq B_{m}\right.$ for all $1 \leq m \leq n+2$ and $\bigcap_{m=1}^{n+2} F_{m, i}=$ $\emptyset$ and $\left.\bigcup_{m=1}^{n+2} F_{m, i}=Y\right\} \in \mathcal{D}$. For any $i$ in this set, then, we have witnesses for the fact that $\operatorname{dim}(Y) \leq n$. $\dashv$

A continuous map between compacta is called weakly confluent in the literature (see [22]) if every subcontinuum in the range is the image of a subcontinuum in the domain. It follows from 2.4, plus the fact that connectedness is preserved by ultracopowers and continuous images, that co-existential maps are weakly confluent. There are many related notions discussed in [22], and weak confluency is the most general. For example, confluency in a mapping says that each component of the pre-image of a subcontinuum maps onto the subcontinuum. We do not know whether there is a direct relation between co-existential maps and confluent maps, but suspect not.

The strongest notion along these lines is monotonicity. A continuous map is called monotone if the pre-images of points under that map are connected sets. In [6] it 
is shown that co-elementary maps onto locally connected compacta are monotone; actually this remains true for co-existential maps.

2.7. Theorem. Let $f: X \rightarrow Y$ be a co-existential map between compacta. If $Y$ is locally connected, then $f$ is monotone.

Proof. Let $y \in Y$ be given, and let $\mathcal{U}$ be a neighborhood basis for $y$ consisting of connected open sets. For each subcontinuum $\bar{U}, U \in \mathcal{U}$, let $f^{*}(\bar{U})$ be the subcontinuum of $X$ guaranteed to exist by $2.4\left(f^{*}(\bar{U})\right.$ being connected by virtue of being a continuous image of an ultracopower of $\bar{U})$. Since $f^{-1}[U] \subseteq f^{*}(\bar{U})$, we have $f^{-1}[\{y\}] \subseteq f^{*}(\bar{U})$. Now $\mathcal{U}$ is a directed set under the ordering of reverse inclusion. Moreover, by $2.4, f^{*}$ is a $\cup$-semilattice homomorphism, hence order preserving. Thus the family $\mathcal{V}:=\left\{f^{*}(\bar{U}): U \in \mathcal{U}\right\}$ is also directed under reverse inclusion. Since $\cap \mathcal{U}=\{y\}$, it follows that $\cap \mathcal{V}=f^{-1}[\{y\}]$. Directed intersections of subcontinua are subcontinua (see [28]); consequently $f^{-1}[\{y\}]$ is connected. $\dashv$

The next result, an easy application of our methods, deals with isolated points.

2.8. Proposition. Let $f: X \rightarrow Y$ be a co-existential map between compacta.

(i) If $y$ is an isolated point of $Y$, then $f^{-1}[\{y\}]$ is an open singleton in $X$.

(ii) If $x$ is an isolated point of $X$ and $Y$ is locally connected, then $f(x)$ is an isolated point of $Y$.

Proof. To prove $(i)$, let $y \in Y$ be isolated. Letting $K$ be $\{y\}$, the corresponding set $f^{*}(K)$ guaranteed by 2.4 must be a singleton open set, and must therefore be the pre-image under $f$ of $y$.

To prove $(i i)$, note that $f^{-1}[\{f(x)\}]$ is connected by 2.7. Since $\{x\}$ is open in $X$, we have $f^{-1}[\{f(x)\}]=\{x\} . \quad\{f(x)\}$ is therefore open since continuous surjections between compacta are quotient maps. $\dashv$

The question naturally arises as to the relationship between co-existential maps and co-elementary maps; as yet we have no examples of co-existential maps that are not co-elementary. To remedy the situation, let us define an arc to be any homeomorphic copy of the closed unit interval in the real line. Arcs are of central importance in the study of compacta (especially continua); they can be characterized as being metrizable continua with exactly two noncut points (a classic result of R. L. Moore). In [3] it is shown (using another classic result of Moore) that any Peano continuum (i.e., locally connected metrizable continuum) is an arc, as long as it is co-elementarily equivalent to an arc. It follows immediately that co-elementary images of arcs are arcs. We can even do a little better. 
2.9. Proposition. Co-existential images of arcs are arcs.

Proof. Let $f: X \rightarrow Y$ be a co-existential map between continua, where $X$ is an arc. $X$ is locally connected; hence so is $Y$. By 2.7, then, $f$ is monotone. Now $Y$ is an infinite metrizable continuum. So the monotonicity of $f$, together with the fact that $X$ is an arc, tells us that $Y$ has exactly two noncut points. Hence $Y$ is an arc. $\dashv$

The main result of [6] is that any monotone continuous surjection between two arcs is a co-elementary map. This gives us the following.

2.10. Theorem. If $f: X \rightarrow Y$ is a co-existential map between compacta, and if $X$ is an arc, then $f$ is co-elementary.

Proof. $Y$ is an arc by $2.9, f$ is monotone by $2.7 . f$ is therefore co-elementary by the main result (Proposition 2.7) of [6]. $\dashv$

2.11. Proposition. If $f: X \rightarrow Y$ is a co-elementary map between compacta, and if $X$ is an arc, then for any subcontinuum $C$ of $X, f \mid C$ is co-elementary onto its image if and only if $C$ and $f[C]$ have the same cardinality.

Proof. In the case $C$ is a singleton, there is no problem. So suppose $C$ is nondegenerate. Then $C$ is an arc. If $f[C]$ is a singleton, then co-elementarity fails of course. Otherwise, $f[C]$ is an arc too. $f \mid C: C \rightarrow f[C]$ is clearly a monotone continuous surjection, and is therefore co-elementary by Proposition 2.7 of [6]. $\dashv$

Co-elementary equivalence preserves covering dimension by results of [2]. Thus any example of a co-existential map that changes dimension is an example of a coexistential map that is not co-elementary. (Of course, by 2.6, a co-existential map that changes dimension must necessarily lower it.)

2.12. Example. We construct a dimension-lowering co-existential map $f: X \rightarrow Y$ between locally connected metrizable continua as follows.

Set $X:=([0,1 / 2] \times\{0\}) \cup[1 / 2,1]^{2}, Y:=[0,1 / 2] \times\{0\}, Z:=[0,1] \times\{0\}$, all subsets of the euclidean plane. $Y$ and $Z$ are $\operatorname{arcs} ; X$ is a "kite-with-tail." $Y$ is a subcontinuum of $X$ (resp., $Z$ ), and we simply let $f$ (resp., $g$ ) retract $X$ (resp., $Z$ ) onto $Y$, collapsing the square (resp., the interval $[1 / 2,1] \times\{0\}$ ) to the point $\langle 1 / 2,0\rangle$. Since $Y$ and $Z$ are arcs, and $g$ is a monotone continuous surjection, we know that $g$ is co-elementary by Proposition 2.7 of [6]. We now let $h$ map $Z$ to $X$ by leaving each element of $[0,1 / 2] \times\{0\}$ fixed and taking $[1 / 2,1] \times\{0\}$ continuously onto $[1 / 2,1]^{2}$ in such a way that $\langle 1 / 2,0\rangle$ remains fixed. Clearly $f h=g$, and $f$ is therefore a co-existential map. 


\section{An Analog of the Löwenheim-Skolem Theorem (Sharper Version).}

Of the many assertions that lie under the rubric "Löwenheim-Skolem," the one with the most "algebraic" phrasing takes on the form of a factorization theorem: Let $\mathcal{L}$ be a first-order lexicon, with $f: A \rightarrow B$ an embedding of $\mathcal{L}$-structures. If $\kappa$ is an infinite cardinal number such that $|A|+|\mathcal{L}| \leq \kappa \leq|B|$, then there exists an $\mathcal{L}$-structure $C$ and embeddings $g: A \rightarrow C, h: C \rightarrow B$ such that $|C|=\kappa, h$ is an elementary embedding and $f=h g$.

For a dualized version of this in the compact Hausdorff setting, we must eliminate reference to a first-order lexicon, as well as decide what cardinal invariant of compacta is to take the place of the underlying-set cardinality. If we take a clue from Stone duality, noting that the weight of a Boolean space equals the cardinality of its clopen algebra, then we must decide upon weight as the invariant of choice. (There are, of course, other reasons to choose weight, but the one given above is the most accessible.) We denote the weight of $X$ by $w(X)$.

3.1. Theorem. Let $f: X \rightarrow Y$ be a continuous surjection between compacta, with $\kappa$ an infinite cardinal such that $w(Y) \leq \kappa \leq w(X)$. Then there is a compactum $Z$ and continuous surjections $g: X \rightarrow Z, h: Z \rightarrow Y$ such that $w(Z)=\kappa, g$ is a co-elementary map, and $f=h g$.

Proof. By way of a preliminary comment, this is a sharper version of the LöwenheimSkolem theorem proved in [4]. In that result we used the model theory of lattices to obtain $Z$ such that $w(Z) \leq \kappa$. There is a bit more work involved in making sure that $w(Z)$ may be any prescribed cardinal in the interval of possibilities.

There is no loss of generality in assuming that $Y$ is infinite; so first assume that $\kappa=w(Y)$, and let $\mathcal{B} \subseteq F(Y)$ be a lattice base of cardinality $\kappa$. Let $\varphi:=f^{F} \mid \mathcal{B}$. Then we may treat $S(\mathcal{B})$ as $Y$ and $\varphi^{S}$ as $f$. By the usual Löwenheim-Skolem theorem, there is a normal disjunctive lattice $A$ and lattice embeddings $\psi: \mathcal{B} \rightarrow A, \theta: A \rightarrow F(X)$ such that $|A|=\kappa, \theta$ is an elementary embedding, and $\varphi=\theta \psi$. Set $Z:=S(A)$, $g:=\theta^{S}$, and $h:=\psi^{S}$. Then $g$ is a co-elementary map, $w(Z) \leq|A|=\kappa$, and $h g=(\theta \psi)^{S}=\varphi^{S}=f$. (Note: This much was proved in [3].) Now $h$ maps $Z$ onto $Y$; and, since continuous surjections between compacta cannot raise weight, we infer that $w(Z)=\kappa$.

The rest of the argument is not model-theoretic at all. Suppose we could factor $f$ into continuous surjections $g: X \rightarrow Z, h: Z \rightarrow Y$, where $w(Z)=\kappa$. Then we could apply the argument in the last paragraph to factor $g$, and we would be done.

To effect this factorization $(f=h g)$, we use the Gel'fand-Naŭmark duality theorem between $\mathbf{C H}$ and the category CBA of commutative (Banach) $B^{*}$-algebras and nonexpansive linear maps. (Recall [26] that a $B^{*}$-algebra is a complex Banach algebra with a unary operation ( $)^{*}$ satisfying: $(a+b)^{*}=a^{*}+b^{*} ;(a b)^{*}=b^{*} a^{*} ;(\lambda a)^{*}=\bar{\lambda} a^{*}$ ( $\lambda$ is a complex scalar, $\bar{\lambda}$ is the complex conjugate of $\lambda$ ); $\left\|a^{*}\right\|=\|a\|$; and $a^{* *}=a$.) For $X \in \mathbf{C H}, C(X)$ is the $B^{*}$-algebra of continuous complex-valued functions on $X$; the norm is the supremum norm, and the involution ()$^{*}$ is defined pointwise by 
$\varphi^{*}(x):=\overline{\varphi(x)}$. The other half of the duality is the maximal ideal space construction $M()$, restricted to objects in CBA.

Claim 1: $w(X)=d(C(X))$, where $d$ is the density, the least cardinality of a dense subset of a topological space. To see this, let $\mathcal{U}$ be the collection of open disks with rational centers and rational radii in the complex plane, and suppose $\Phi \subseteq C(X)$ is a dense subset. Set $\mathcal{V}:=\left\{\varphi^{-1}[U]: \varphi \in \Phi, U \in \mathcal{U}\right\}$. $\mathcal{V}$ is an open base for $X$. Indeed, if $W$ is an open neighborhood of $x$ in $X$, let $\psi: X \rightarrow[0,1]$ take $x$ to 0 and $X \backslash W$ to 1 . Let $\varphi \in \Phi$ be such that $\|\varphi-\psi\|<1 / 4$. Then $\|\varphi(0)\|<1 / 4$ and $\|\varphi(y)\|>3 / 4$ for $y \in X \backslash W$; hence if $U \in \mathcal{U}$ is the open disk of radius $1 / 2$ centered at the origin, then $\varphi^{-1}[U]$ is a set in $\mathcal{V}$ containing $x$ and contained in $W$. Since $|\mathcal{V}| \leq|\Phi|$, we have $w(X) \leq d(C(X))$. Now let $\mathcal{V}$ be an open base for $X$. By Weierstrass approximation, we can get a dense $\Phi \subseteq C(X)$ of cardinality $\leq|\mathcal{V}|$. Thus we have $d(C(X)) \leq w(X)$.

Claim 2: There is a subset $\Phi$ of $C(X)$, consisting of maps into the unit interval, such that: $(i)|\Phi|=w(X)$; and $(i i)\|\varphi-\psi\| \geq 1 / 2$ for all $\varphi, \psi \in \Phi$. To see this, first note that any set $\Phi$ satisfying (ii) must have cardinality at most $d(C(X))$ (which is $w(X)$, by Claim 1). Now let $\Phi$ be maximal with regard to satisfying (ii), let $U$ be the open disk of radius $1 / 2$ and centered at the origin in the complex plane, and set $\mathcal{V}:=\left\{\varphi^{-1}[U]: \varphi \in \Phi\right\}$. We need to show $\mathcal{V}$ is an open base for $X$. Assuming the contrary, there exist $x \in W \subseteq X, W$ open in $X$, such that for all $\varphi \in \Phi$, if $\varphi(x) \in U($ so $\varphi(x) \in[0,1 / 2))$, then there is some $y \notin W$ with $\varphi(y) \in[0,1 / 2)$. Let $\psi: X \rightarrow[0,1]$ take $x$ to 0 and $X \backslash W$ to 1 . If $\varphi \in \Phi$ and $\varphi(x) \in[1 / 2,1]$, then $\|\psi-\varphi\| \geq|\psi(x)-\varphi(x)| \geq 1 / 2$. If $\varphi(x) \in[0,1 / 2)$, let $y \in X \backslash W$ be such that $\varphi(y) \in[0,1 / 2)$. Then $\|\psi-\varphi\| \geq|\psi(y)-\varphi(y)| \geq 1 / 2$. Thus $\Phi \cup\{\psi\}$ properly contains $\Phi$ and satisfies $(i i)$, contradicting the maximality of $\Phi$. Since $\mathcal{V}$ is an open base for $X$, and $|\mathcal{V}| \leq \Phi$, we have $w(X) \leq|\Phi|$. Thus $|\Phi|=w(X)$.

Now we have an embedding $f^{C}: C(Y) \rightarrow C(X)$. Let $\kappa$ be such that $w(Y) \leq$ $\kappa \leq w(X)$, and let $\Phi \subseteq C(X)$ have cardinality $\kappa$ and satisfy (ii) in Claim 2. Let $\Psi \subseteq C(Y)$ be dense of cardinality $w(Y)$. Then $\Phi \cup f^{C}[\Psi]$ is a subset of $C(X)$ of cardinality $\kappa$; closure of this set under the Banach algebra operations with scalar multiplication restricted to the rational complex numbers then results in a subring $A$, also of cardinality $\kappa$. The topological closure $\bar{A}$ of $A$ is therefore a $B^{*}$-subalgebra of $C(X)$. Because $|A|=\kappa, d(\bar{A}) \leq \kappa$. Because $\Phi \subseteq \bar{A}, d(\bar{A}) \geq \kappa$. Because $\Psi$ is dense in $C(Y), f^{C}[C(Y)] \subseteq \bar{A}$. Thus, by applying the maximal ideal functor $M($ ) (i.e., $Z:=M(\bar{A})$ ), we get the factorization we want. This completes the proof. $\dashv$

Let us return, for the moment, to the model-theoretic setting; for simplicity, assume the underlying lexicon $\mathcal{L}$ is countable. A nice application of the Löwenheim-Skolem factorization theorem mentioned in the lead paragraph of this section is the following.

3.2. Proposition. Let $f: A \rightarrow B$ be an embedding between infinite $\mathcal{L}$-structures, with $\kappa \leq|A|$ an infinite cardinal. Suppose that for each $\mathcal{L}$-structure $C$ of cardinality $\kappa$, and each elementary embedding $g: C \rightarrow A$, the composition $f g$ is elementary 
(resp., existential). Then $f$ is elementary (resp., existential).

Proof. Let $\varphi\left(x_{1}, \ldots, x_{n}\right)$ be any (existential) formula, with $\left\langle a_{1}, \ldots, a_{n}\right\rangle$ an $n$-tuple from $A$. By Löwenheim-Skolem, there is an $\mathcal{L}$-structure $C$ of cardinality $\kappa$ and an elementary embedding $g: C \rightarrow A$ such that $g[C]$ contains each $a_{m}$. The desired conclusion is now immediate. $\dashv$

The proof above, while extremely simple, suffers the major failing of being nonportable. The Proposition has an obvious restatement in the language of compacta, and this proof sheds very little light on how to establish the dualized version. There is, however, an argument, based solely on ultraproduct considerations (inspired by C. C. Chang's ultraproduct proof of the compactness theorem, see $[9,11]$ ), which can be easily transported to the topological context. Its drawback is that it is relatively cumbersome. We dualize that argument in the following.

3.3. Theorem. Let $f: X \rightarrow Y$ be a continuous surjection between infinite compacta, with $\kappa \leq w(Y)$ an infinite cardinal. Suppose that for each compactum $Z$ of weight $\kappa$, and each co-elementary map $g: Y \rightarrow Z$, the composition $g f$ is co-elementary (resp., co-existential). Then $f$ is co-elementary (resp., co-existential).

Proof. In model theory, the obstruction to being able to show, with mapping diagrams, that the class of elementary (resp., existential) embeddings is closed under terminal factors, resides in a failure of surjectivity: one cannot carry out a successful diagram chase. On the topological side, the obstruction to closure under initial factors resides in a failure of injectivity. Both these failures can be remedied somewhat with the use of ultra(co)products.

We establish our result for the co-elementary case; the co-existential case is handled in like fashion. First let $\Delta$ be the set of finite subsets of $Y$. For each $\delta \in \Delta$ there is a continuous mapping $r_{\delta}$ from $Y$ to the closed unit interval, such that the restriction $r_{\delta} \mid \delta$ is one-one. Let $W_{\delta}:=r_{\delta}[Y]$. By 3.1, there is a compactum $Z_{\delta}$ of weight $\kappa$, and continuous surjections $g_{\delta}: Y \rightarrow Z_{\delta}, t_{\delta}: Z_{\delta} \rightarrow W_{\delta}$, such that $g_{\delta}$ is co-elementary and $r_{\delta}=t_{\delta} g_{\delta}$. So each $g_{\delta}$ is a co-elementary map onto a compactum of weight $\kappa$, and $g_{\delta} \mid \delta$ is one-one.

Now, with the aid of 2.1, we build a diagram $\mathbf{D}_{\delta}$ that witnesses the co-elementarity above, for each $\delta \in \Delta$. More precisely, we have a single ultrafilter $\mathcal{D}$ on a set $I$, and homeomorphisms $h_{\delta}: X I \backslash \mathcal{D} \rightarrow Z_{\delta} I \backslash \mathcal{D}, k_{\delta}: Y I \backslash \mathcal{D} \rightarrow Z_{\delta} I \backslash \mathcal{D}$ witnessing the coelementarity of $g_{\delta} f$ and $g_{\delta}$ respectively. Letting $p_{\delta}: Z_{\delta} I \backslash \mathcal{D} \rightarrow Z_{\delta}, p_{X}: X I \backslash \mathcal{D} \rightarrow X$, and $p_{Y}: Y I \backslash \mathcal{D} \rightarrow Y$ be the canonical codiagonal maps, we then have $p_{\delta} k_{\delta}=g_{\delta} p_{Y}$ and $p_{\delta} h_{\delta}=g_{\delta} f p_{X}$.

In general we cannot expect $f p_{X}=p_{Y} k_{\delta}^{-1} h_{\delta}$; however it is true that $g_{\delta} f p_{X}=$ $g_{\delta} p_{Y} k_{\delta}^{-1} h_{\delta}$. We now proceed to take an "ultracoproduct" of the diagrams $\mathbf{D}_{\delta}$.

For each $\delta \in \Delta$, let $\hat{\delta}:=\{\gamma \in \Delta: \delta \subseteq \gamma\}$. Then the set $\{\hat{\delta}: \delta \in \Delta\}$ clearly satisfies the finite intersection property, and hence extends to an ultrafilter $\mathcal{H}$ on $\Delta$. Form the "H-H-ultracoproduct" diagram $\mathbf{D}$ in the obvious way. Then we have the 
canonical codiagonal maps $u_{X}: X \Delta \backslash \mathcal{H} \rightarrow X, v_{X}:(X I \backslash \mathcal{D}) \Delta \backslash \mathcal{H} \rightarrow X I \backslash \mathcal{D}, u_{Y}:$ $Y \Delta \backslash \mathcal{H} \rightarrow Y$, and $v_{Y}:(Y I \backslash \mathcal{D}) \Delta \backslash \mathcal{H} \rightarrow Y I \backslash \mathcal{D}$. Moreover, $p_{X} v_{X}=u_{X}\left(p_{X} \Delta \backslash \mathcal{H}\right)$ and $p_{Y} v_{Y}=u_{Y}\left(p_{Y} \Delta \backslash \mathcal{H}\right)$ are (essentially) codiagonal maps from iterated ultracopowers, and these ultracopowers are isomorphic via $\left(\sum_{\mathcal{H}} k_{\delta}\right)^{-1}\left(\sum_{\mathcal{H}} h_{\delta}\right)=\sum_{\mathcal{H}} k_{\delta}^{-1} h_{\delta}$.

We need to show that $f p_{X} v_{X}=p_{Y} v_{Y}\left(\sum_{\mathcal{H}} k_{\delta}\right)^{-1}\left(\sum_{\mathcal{H}} h_{\delta}\right)$. Suppose otherwise. Then we have some $x \in(X I \backslash \mathcal{D}) \Delta \backslash \mathcal{H}$ with $\left[f p_{X} v_{X}\right](x)=y_{1} \neq y_{2}=\left[p_{Y} v_{Y}\left(\sum_{\mathcal{H}} k_{\delta}\right)^{-1}\left(\sum_{\mathcal{H}} h_{\delta}\right)\right](x)$. Because $\mathcal{H}$ is an ultrafilter, we have $\left\{\gamma \in \Delta: v_{Y}\left(\sum_{\mathcal{H}} k_{\delta}\right)^{-1}\left(\sum_{\mathcal{H}} h_{\delta}\right)=k_{\gamma}^{-1} h_{\gamma} v_{X}\right\} \in \mathcal{H}$; hence $\left\{\gamma \in \Delta: y_{2}=\left[p_{Y} k_{\gamma}^{-1} h_{\gamma} v_{X}\right](x)\right\} \in \mathcal{H}$. Now $\left[g_{\gamma} p_{Y} k_{\gamma}^{-1} h_{\gamma} v_{X}\right](x)=\left[g_{\gamma} f p_{X} v_{X}\right](x)$ for all $\gamma \in \Delta$, so $\left\{\gamma \in \Delta: g_{\gamma}\left(y_{2}\right)=g_{\gamma}\left(y_{1}\right)\right\} \in \mathcal{H}$. However, $\left\{\gamma \in \Delta: g_{\gamma}\left(y_{2}\right) \neq\right.$ $\left.g_{\gamma}\left(y_{1}\right)\right\} \supseteq\left\{\widehat{y_{1}, y_{2}}\right\} \in \mathcal{H}$. This contradiction completes the proof. $\dashv$

\section{An Analog of the Elementary Chains Theorem.}

The simplest version of the elementary chains theorem, due jointly to A. Tarski and R. L. Vaught, says that the union of an $\omega$-indexed elementary chain of relational structures is an elementary extension of each of its summands. The obvious translation into the realm of compacta, what we refer to here as the co-elementary chains hypothesis $(\mathrm{CECH})$ is the assertion that the inverse limit of an $\omega$-indexed co-elementary chain of compacta is a co-elementary cover of each of its factors. More precisely, if $\left\langle X_{n} \stackrel{f_{n}}{\leftarrow} X_{n+1}: n<\omega\right\rangle$ is an $\omega$-indexed family of co-elementary maps, and if $X:=\lim X_{n}$ is the inverse limit space with natural connecting maps $g_{n}: X \rightarrow X_{n}$, $n<\omega$, then each $g_{n}$ is a co-elementary map. (Recall that $X$ is defined to be the subspace $\left\{\left\langle x_{0}, x_{1}, \ldots\right\rangle \in \prod_{n<\omega} X_{n}: x_{n}=f_{n+1}\left(x_{n+1}\right)\right.$ for all $\left.n<\omega\right\}$, and $g_{n}$ is projection to the $n$th factor, restricted to this subspace.)

The way one normally goes about proving the usual elementary chains theorem is to use induction on the complexity of formulas. We know of no other way; in particular, we know of no way to prove the result using the ultrapower theorem and chains of isomorphisms of ultrapowers. This perhaps speaks to an inherent lack of "fine structure" in ultraproduct methods and definitely presents difficulties when one tries to find a direct proof of the $\mathrm{CECH}$. While the $\mathrm{CECH}$ is true, the only proof we know of uses Gel'fand-Naimark duality and Banach model theory (see below).

The following weak version of the CECH can be proved by direct methods (avoiding Banach model theory, at any rate), and is worth while exploring. The difference between the weak and strong versions of the $\mathrm{CECH}$ may be compared to the difference between the amalgamation property for elementary embeddings (resp., co-elementary maps) and the existence of elementary pushouts (resp., co-elementary pullbacks).

4.1. Proposition. Let $\left\langle X_{n} \stackrel{f_{n}}{\longleftarrow} X_{n+1}: n<\omega\right\rangle$ be a co-elementary chain of compacta. Then there exists a compactum $X$ and co-elementary maps $g_{n}: X \rightarrow X_{n}, n<\omega$, such that for all $n<\omega, g_{n}=f_{n} g_{n+1}$. 
Proof. By 2.1, there is an ultrafilter $\mathcal{D}$ on a set $I$ and homeomorphisms $X_{n} I \backslash \mathcal{D} \stackrel{h_{n}}{\longleftarrow}$ $X_{n+1} I \backslash \mathcal{D}, n<\omega$, such that for each $n, p_{X_{n}, \mathcal{D}} h_{n}=f_{n} p_{X_{n+1}, \mathcal{D}}$. Let $X$ be the inverse limit of this chain, with natural maps $k_{n}: X \rightarrow X_{n} I \backslash \mathcal{D}$. Since each $h_{n}$ is a homeomorphism, so is each $k_{n}$, and we set $g_{n}:=p_{X_{n}, \mathcal{D}} k_{n}$. $\dashv$

Of course the weakness of our weak version of the CECH in 4.1 lies in the fact that the compactum $X$ is not generally $\lim X_{n}$. This would be remedied if we could show that the natural map $h: X \rightarrow \underset{\leftarrow}{\leftarrow} \leftarrow X_{n}$, a continuous surjection, is actually co-elementary; but that seems to be beyond our methods.

The only way we know of to prove the CECH is to use Banach model theory. For reasons of space, our argument is far from self-contained; the interested reader is referred to the appropriate literature (e.g., $[14,15,16,17,18]$ ) for details. The skeleton of the approach is this: The basic lexicon of Banach model theory uses the usual symbols from the theory of abelian groups, plus countably many unary operation symbols to allow for scalar multiplication. (The scalar field can be either the field of real rationals or complex rationals.) Also there is a unary predicate symbol whose intended interpretation is the closed unit ball in a Banach space. (The theory is flexible enough to allow for additional operation symbols; e.g., lattice symbols, multiplication and involution.)

Banach model theory does not use all first-order formulas, just the positive-bounded ones (i.e., conjunction, disjunction, and quantification restricted to the unit ball). Also the relation of satisfaction is weakened to what is called approximate satisfaction. Once this is all laid out, the notions of elementary equivalence and elementary embedding make sense in the Banach setting. Finally there is an analogous ultrapower theorem, where "ultrapower" means Banach ultrapower and "isomorphic" means isometrically isomorphic. This tells us that the Banach notion of elementary embedding is the exact analog of our notion of co-elementary map.

We now come to the main bridge connecting the Banach world and the world of compacta, namely the Gel'fand-Nămark duality, a genuine category-theoretic duality between $\mathbf{C H}$ and the category CBA of commutative $B^{*}$-algebras and nonexpansive linear maps (see the proof of 3.1 above). Using a result called the perturbation lemma [17], the obvious analog of the classic elementary chains theorem is proved in the Banach setting [18]. (Connecting maps are elementary embeddings in the Banach sense, and the full direct limit is the direct limit in the category CBA when the summands happen to be commutative $B^{*}$-algebras.) So the $\mathrm{CECH}$ is nothing but the Gel'fandNaImark dual of the Banach elementary chains theorem restricted to objects in CBA. We thus have what is essentially a proof of the $\mathrm{CECH}$.

4.2. Theorem. In 4.1, $X$ may be taken to be the inverse $\operatorname{limit} \lim _{\leftarrow} X_{n}$.

4.3. Remark. The technique for proving the elementary chains theorem allows for generalization to directed systems of elementary embeddings. This then gives us a 
corresponding generalized CECH.

\section{An Analog of Robinson's Test.}

The notion of model completeness in first-order model theory was invented by A. Robinson, who was inspired by classical (Nineteenth-Century) algebra; in particular, Hilbert's Nullstellensatz concerning the location of solutions of systems of equations and inequations. (See, e.g., [19, 21].) One of many equivalent formulations of this notion is to say that a first-order theory is model complete if any embedding between two models of the theory is elementary. Since elementary embeddings are a great deal rarer than embeddings in general, it is very interesting when a first-order theory is discovered to be model complete. (Examples include (see [9]): dense linear orderings without endpoints; atomless Boolean algebras; algebraically closed fields; real closed fields; real closed ordered fields.) It is not surprising, then, that much effort has been expended in the study of this phenomenon; especially in the search for readily applicable tests to detect its presence.

Robinson's test says that a theory is model complete just in case every embedding between models of the theory is an existential embedding. Since testing for existentiality is ostensibly easier than testing for elementarity, this result is very important in the general study (as well as being a strikingly elegant application of the elementary chains theorem).

To give Robinson's test a proper phrasing in the compact Hausdorff setting, we first define a class of compacta to be co-elementary if it is closed under co-elementary equivalence and the taking of ultracoproducts. (This corresponds precisely to a class of relational structures being the class of models of a first-order theory; examples include (see [2]): Boolean spaces without isolated points; continua; (in)decomposable continua; compacta of covering dimension $n, n<\omega$; infinite-dimensional compacta. The class of locally connected compacta is not co-elementary $[13,5]$.) A co-elementary class is model cocomplete if any continuous surjection between two of its members is a co-elementary map. (Aside: recalling what completeness means in model theory, the right criterion for a co-elementary class to be cocomplete is that it consist of just one co-elementary equivalence class. It is not entirely trivial to show that co-elementary equivalence classes are indeed co-elementary classes, but true nonetheless. (See [7]. Lemma 2.1 greatly facilitates the proof that co-elementary equivalence classes are closed under ultracoproducts.) The notions of (co)completeness and model (co)completeness, while cognate, are not directly related logically. An obvious analog of the prime model test, also obviously true in the topological setting, is that a model cocomplete co-elementary class $\mathbf{K}$ is cocomplete if there is some $X \in \mathbf{K}$ such that every member of $\mathbf{K}$ continuously surjects onto $X$.) It is our belief that the reasons for studying model completeness in model theory remain just as compelling when we consider the topological analog; the present work is just a beginning of a process of discovering interesting co-elementary classes that are model cocomplete. 
5.1. Theorem. A co-elementary class of compacta is model cocomplete if and only if every continuous surjection in the class is a co-existential map.

Proof. Our proof is an exact dualization of the well-known proof of Robinson's test using elementary chains (see, e.g., [21]); we present it here for the sake of thematic completeness.

Only one direction is nontrivial. Assume that $\mathbf{K}$ is a co-elementary class with the property that every continuous surjection in $\mathbf{K}$ is a co-existential map, and let $f: X \rightarrow Y$ be a continuous surjection in $\mathbf{K} . f$ is co-existential, so we get a compactum $Z_{1}$ and continuous surjections $g_{0}: Z_{1} \rightarrow Y, h_{0}: Z_{1} \rightarrow X$ with $g_{0}$ co-elementary and $g_{0}=f h_{0} . Z_{1}$ is thus in $\mathbf{K}$, and we conclude that $h_{0}$ is co-existential. Repeat the procedure. We get a compactum $Z_{2}$ and continuous surjections $g_{1}: Z_{2} \rightarrow X, h_{1}: Z_{2} \rightarrow Z_{1}$ with $g_{1}$ co-elementary and $g_{1}=h_{0} h_{1} . Z_{2} \in \mathbf{K}$, and $h_{1}$ is thus a co-existential map. Proceeding in this way, we obtain compacta $Z_{n} \in \mathbf{K}$ and continuous surjections $g_{n}, h_{n}$, $n<\omega$. For $n \geq 1, h_{n}: Z_{n+1} \rightarrow Z_{n}, g_{n+1}: Z_{n+2} \rightarrow Z_{n}, g_{n+1}=h_{n} h_{n+1}$. Each $h_{n}$ is co-existential, each $g_{n}$ is co-elementary. The inverse limit $Z$ of the maps $h_{n}, n<\omega$, is also the inverse limit of the co-elementary maps $g_{n}, n$ odd (resp., $n$ even). By the CECH (4.2), there are co-elementary maps $u: Z \rightarrow X$ and $v: Z \rightarrow Y$ such that $v=f u$. Since the class of co-elementary maps is closed under terminal factors, it follows that $f$ is co-elementary. $\dashv$

There is a sharper version of Robinson's test (see, e.g., [9]). One assumes that the theory has no finite models; and that, for some infinite cardinal $\kappa \geq|\mathcal{L}|$, any embedding between models of cardinality $\kappa$ is an existential embedding. Then the theory is model complete. The following is the topological analog of this fact.

5.2. Theorem. Let $\mathbf{K}$ be a co-elementary class containing no finite compacta. Then $\mathbf{K}$ is model cocomplete if (and only if) there is some infinite cardinal $\kappa$ such that each continuous surjection between compacta of weight $\kappa$ in $\mathbf{K}$ is a co-existential map.

Proof. Let $f: X \rightarrow Y$ be a continuous surjection in $\mathbf{K}$. By 5.1, it suffices to show that $f$ is co-existential. Assume first that $\kappa \leq w(Y)$. By 3.3, it suffices to show that

$g f$ is co-existential whenever $g: Y \rightarrow Z$ is co-elementary and $w(Z)=\kappa$. So let $g$ be given. By 3.1, we can obtain a factorization $u: X \rightarrow W, v: W \rightarrow Z$, where $u$ is co-elementary, $w(W)=w(Z)=\kappa$, and $g f=v u$. Of course both $W$ and $Z$ are in $\mathbf{K}$, so $v$ is co-existential. Then $v u$ is co-existential by 2.2 .

If $\kappa>w(Y)$, then find an ultrafilter $\mathcal{D}$ on a set $I$ such that $w(Y I \backslash \mathcal{D}) \geq \kappa$ (see $[2])$. By the argument above, we conclude that $f I \backslash \mathcal{D}$ is co-existential. Immediately we infer (see 2.2) that $f$ is co-existential. $\dashv$

\section{Co-inductive Co-elementary Classes.}

The usual elementary chains theorem shows that any model complete theory is inductive; i.e., closed under chain unions (indeed, direct limits of directed systems 
of embeddings). Being inductive, by the Chang-Łoś-Suszko theorem, is equivalent to being AE axiomatizable. A co-elementary class $\mathbf{K}$ is co-inductive if it is closed under inverse limits of directed systems of continuous surjections. Because of 4.2, we know that model cocomplete co-elementary classes are co-inductive.

The following notion dualizes that of being existentially closed relative to an elementary class of relational structures. Let $\mathbf{K}$ be a co-elementary class, with $X \in \mathbf{K}$. $X$ is co-existentially closed in $\mathbf{K}$ if whenever $f: Y \rightarrow X$ is a continuous surjection and $Y \in \mathbf{K}$, then $f$ is a co-existential map.

6.1. Theorem. Let $\mathbf{K}$ be a co-inductive co-elementary class, with $X \in \mathbf{K}$ infinite. Then $X$ is the continuous image of some $Y$ that is co-existentially closed in $\mathbf{K}$, such that $w(Y)=w(X)$.

Proof. This is the dualization of a well-known property of inductive elementary classes, so we supply just the transition steps.

The class of normal disjunctive lattices whose maximal spectra lie in $\mathbf{K}$ is suitably denoted $S^{-1}[\mathbf{K}]$. The class NDL of normal disjunctive lattices is itself an inductive elementary class. Since $S($ ) converts direct limits to inverse limits, we conclude that $S^{-1}[\mathbf{K}]$ is an inductive elementary class whenever $\mathbf{K}$ is a co-inductive co-elementary class. Suppose $X \in \mathbf{K}$ is infinite, and pick a lattice base $\mathcal{A}$ for $X$ of infinite cardinality $w(X)$. Then $\mathcal{A} \in S^{-1}[\mathbf{K}]$, and there is an embedding $f: \mathcal{A} \rightarrow B$ for some existentially closed $B \in S^{-1}[\mathbf{K}]$ such that $|B|=|\mathcal{A}|$. (The proof of this is elementary model theory, involving unions of chains of embeddings (see $[9,19])$.) Set $Y:=S(B)$. Then $f^{S}$ is (essentially) a continuous surjection from $Y$ to $X$, so $w(X) \leq w(Y)$. But $w(Y) \leq|B|=|\mathcal{A}|=w(X)$, so the weights are equal. Finally, if $g: Z \rightarrow Y$ is a continuous surjection, $Z \in \mathbf{K}$, then $g^{F}: F(Y) \rightarrow F(Z)$ is an embedding in $S^{-1}[\mathbf{K}]$. Let $u: B \rightarrow F(Y)$ be the obvious separative embedding. Since $B$ is existentially closed, $g^{F} u$ is an existential embedding. Since $S($ ) converts existential embeddings to coexistential maps (and separative embeddings to homeomorphisms), we infer that $g$ is a co-existential map. $\dashv$

By way of a linguistic aside, suppose "blob" is a noun given to name a class of compacta that is a co-inductive co-elementary class. Then we may abbreviate " $X$ is co-existentially closed in the class of blobs" as " $X$ is a co-existentially closed blob." Note, however, that this does not mean that $X$ is a blob that is co-existentially closed in the class of compacta, any more than " $G$ is a free abelian group" means that $G$ is a free group that happens to be abelian. (Or, more prosaically, any more than "George is a small elephant" means that George is a small entity that happens to be an elephant.) (W. V. O. Quine [23] applies the word syncategorematic to such adjectives as "co-existentially closed," "free," and "small" used in this way.) Because of 6.1, we know that there exist co-existentially closed compacta in all infinite weights. The same can be said for the existence of co-existentially closed continua, since the class of continua is co-inductive co-elementary. Characterizing the co-existentially closed blobs, then, is a new problem each time we change the meaning of the word "blob." 
When this meaning is least restrictive, the problem turns out to have an easy solution.

6.2. Proposition. The co-existentially closed compacta are precisely the Boolean spaces without isolated points.

Proof. We call a space without isolated points self-dense. Suppose $X$ is a coexistentially closed compactum. Then $X$ is a co-existential image of a Stone-Cech compactification $\beta(I)$ for a suitably large discrete space $I$. By 2.5(iii), $X$ must therefore be Boolean. By Stone duality, its clopen algebra $B(X)$ must be an existentially closed Boolean algebra. Now the class of such algebras comprises the atomless Boolean algebras (see [21]); hence $X$ is a self-dense Boolean space.

In the other direction, suppose $X$ is a self-dense Boolean space, with $f: Y \rightarrow X$ a continuous surjection between compacta. Let $I$ be a discrete space of cardinality $|Y|$, and set $Z:=\beta(I) \times W$, where $W$ is any self-dense Boolean space. Then $Z$ is a self-dense Boolean space, and there is a continuous surjection $g: Z \rightarrow Y$. Let $h:=f g$. Then $h$ is a continuous surjection between self-dense Boolean spaces; hence $h^{B}: B(X) \rightarrow B(Z)$ is an embedding between atomless Boolean algebras. The class of such algebras is model complete; therefore $h^{B}$ is elementary. This says that $h$ is a co-elementary map, proving that $f$ is co-existential. $\dashv$

The problem of identifying the co-existentially closed continua is still open, and seems to be quite difficult. What little we know so far, besides the fact that they exist in all infinite weights, is the following.

6.3. Proposition. Every co-existentially closed continuum is indecomposable. (Consequently, every nondegenerate continuum is a continuous image of an indecomposable continuum of the same weight.)

Proof. Suppose $X$ is a decomposable continuum. Then (see [20, 28]) $X$ has a proper subcontinuum $K$ and a nonempty open set $U \subseteq K$. Pick $x_{0} \in X \backslash K$, and let $Y:=(X \times\{0,1\}) / \sim$ be the quotient space where the only nontrivial identification is $\left\langle x_{0}, 0\right\rangle \sim\left\langle x_{0}, 1\right\rangle$. Let $f: Y \rightarrow X$ be induced by projection onto the first factor. Then $f$ is a continuous surjection between continua, and $f^{-1}[U]$ intersects both components of $f^{-1}[K]$. By 2.4, then, $f$ cannot be a co-existential map and $X$ is therefore not a co-existentially closed continuum. The parenthetical assertion is now an instant corollary of 6.1 and the above. $\dashv$

In model theory, there is an important connection between model completeness and categoricity. In particular, there is Lindström's test (see [19, 21]), which says that if a consistent AE theory $T$ has no finite models and is $\kappa$-categorical for some infinite cardinal $\kappa \geq|\mathcal{L}(T)|$, then $T$ is model complete. The techniques developed here allow us to establish a topological analog of this. First define a co-elementary class $\mathbf{K}$ to be $\kappa$-categorical, where $\kappa$ is an infinite cardinal, if: $(a)$ there are compacta of weight $\kappa$ in $\mathbf{K}$; and (b) any two compacta of weight $\kappa$ in $\mathbf{K}$ are homeomorphic. (Clearly, by the 
Löwenheim-Skolem theorem (3.1), if $\mathbf{K}$ is a co-elementary class containing no finite compacta, and if $\mathbf{K}$ is $\kappa$-categorical for some cardinal $\kappa$, then $\mathbf{K}$ is cocomplete.)

6.4. Theorem. Let $\mathbf{K}$ be a co-elementary class containing no finite compacta. If $\mathbf{K}$ is co-inductive and $\kappa$-categorical for some infinite cardinal $\kappa$, then $\mathbf{K}$ is model cocomplete.

Proof. By 5.2, it suffices to show that every continuous surjection between compacta of weight $\kappa$ in $\mathbf{K}$ is co-existential. But 6.1 and $\kappa$-categoricity tell us that all compacta in $\mathbf{K}$ of weight $\kappa$ are co-existentially closed in $\mathbf{K}$. $\dashv$

6.5. Remarks and Questions. ( $i$ ) Concrete examples of co-elementary and coexistential maps are hard to find. One simple question we would like to settle is the following: Are monotone continuous surjections between nondegenerate locally connected continua necessarily co-existential maps? In particular, is the projection map from the closed unit square onto its first coördinate a co-existential map?

(ii) If $\mathbf{K}$ is a co-elementary class containing at least one infinite compactum, then $S^{-1}[\mathbf{K}]$ is never model complete. By Proposition 2.8 in [7], $f^{F}: F(Y) \rightarrow F(X)$ is an elementary embedding just in case $f: X \rightarrow Y$ is a homeomorphism. The existence of codiagonal maps in $\mathbf{K}$ that are not homeomorphisms then provides embeddings in $S^{-1}[\mathbf{K}]$ that are not elementary.

(iii) Define a compactum $X$ to be $\kappa$-categorical if: $(a)$ the co-elementary equivalence class of $X$ is $\kappa$-categorical; and $(b) w(X)=\kappa . \quad X$ is categorical if $X$ is $w(X)$-categorical. The only categorical compacta we know of are Boolean (the Cantor discontinuum, for example, being $\aleph_{0}$-categorical). By the main result of [3], arcs are "categorical" in a restricted sense: one may look only among the locally connected compacta. Indeed, every infinite compactum is co-elementarily equivalent to a compactum (of any prescribed infinite weight) that is not locally connected [5], so any examples of infinite categorical compacta must fail to be locally connected. In fact, if $X$ is $\kappa$-categorical and $Y \equiv X$ has weight $\geq \kappa$, then $Y$ co-elementarily surjects onto some compactum $Z$ of weight $\kappa$. $Z$ is then homeomorphic to $X$, which is not locally connected. It follows that $Y$ is not locally connected either. In particular, no compactum co-elementarily equivalent to an $\aleph_{0}$-categorical compactum can be locally connected. For any cardinal $\kappa$ (finite too), define a compactum $X$ to be $\kappa$-wide if for each cardinal $\lambda<\kappa, X$ contains a family of $\lambda$ pairwise disjoint proper subcontinua with nonempty interiors. Clearly an infinite locally connected compactum is $\aleph_{1}$-wide; and decomposability for continua is equivalent to being 2-wide. By techniques similar to those used to show that the classes of (in)decomposable continua are co-elementary (see $[5,13]$ ), one can prove that the class of $\aleph_{0}$-wide compacta is co-elementary (but not its complement). (Indeed, for $n<\omega, \sum_{\mathcal{D}} X_{i}$ is $n$-wide if and only if $\left\{i \in I: X_{i}\right.$ is $n$-wide $\} \in \mathcal{D}$.) So if $X$ is co-elementarily equivalent to an infinite locally connected compactum, then $X$ is $\aleph_{0}$-wide. We do not think that the converse is true. (In a private conversation, C. W. Henson has told us that an 
$\aleph_{0}$-categorical compactum must fail to be $\aleph_{0}$-wide. His method uses a Banach version of the Ryll-Nardzewski theorem.)

(iv) We still lack examples of model cocomplete co-elementary classes that are not subclasses of the Boolean spaces.

$(v)$ We lack examples of pairs of co-elementarily equivalent Peano continua that are not homeomorphic.

(vi) What, besides 6.3, can we infer about co-existentially closed continua? (In view of 2.6, for example, we suspect that co-existentially closed continua are curves; i.e., one-dimensional.) Dare we hope for a classically topological classification? Are any of the familiar examples of indecomposable continua (e.g., pseudo-arcs, solenoids) co-existentially closed?

(vii) Let $\mathbf{C C H}$ be the class of continua (considered as a full subcategory of $\mathbf{C H}$ ). There is a single AE sentence $\gamma$ in the first-order language of bounded lattices such that a normal disjunctive lattice $A$ satisfies $\gamma$ if and only if $S(A) \in \mathbf{C C H}$ (see [7]). Let $\mathbf{C N D L}:=\{A \in \mathbf{N D L}: A \models \gamma\}=S^{-1}[\mathbf{C C H}]$. Then, as in the proof of 6.1 , each existentially closed member of CNDL gives rise to a co-existentially closed member of CCH. Is this assignment surjective? Is the class of existentially closed members of CNDL an elementary class? (This deals with the existence of model completions/companions; see [21].) What about the dual question for the class of coexistentially closed members of $\mathbf{C C H}$ ? (For example, if we could characterize the coexistentially closed members of $\mathbf{C C H}$ as the indecomposable curves, a co-elementary class, then the answer to the last question would be yes, and we would have a nice example of a model cocomplete co-elementary class of continua.)

(viii) Two properties closely related to being co-existentially closed in the class of continua are class $(\mathrm{W})$ and $\operatorname{class}(\mathrm{C})$. A continuum $X$ is in $\operatorname{class}(\mathbf{W})$ (resp., in class(C)) if whenever $f: Y \rightarrow X$ is a continuous surjection and $Y$ is a continuum, then $f$ is weakly confluent (resp., confluent). (These notions are due to A. Lelek [22].) Clearly if $X$ is a co-existentially closed continuum, then $X$ is in class(W). Class(W) is definitely broader, since it contains all arcs. Spaces in class(W) are unicoherent; i.e., possessed of the feature that the intersection of any two subcontinua whose union is everything must be connected. There are some nice characterizations of class $(\mathrm{W})$, but they go beyond our scope. An elegant characterization of class $(\mathrm{C})$ is simply being hereditarily indecomposable.

$(i x)$ We would like to see a direct ultracoproduct proof of 4.2, one that doesn't rely on Banach model theory.

[Added in Proof: Re 6.5(vi), co-existentially closed continua are one-dimensional. Re $6.5(i x)$, there is now a proof of 4.2 that involves no Banach model theory. These results will appear in the forthcoming paper [8].]

\section{REFERENCES}

[1] B. Banaschewski, "More on compact Hausdorff spaces and finitary duality," Can. J. Math. 36(1984), 1113-1118. 
[2] P. Bankston, "Reduced coproducts of compact Hausdorff spaces," J. Symbolic Logic 52(1987), 404-424.

[3] — "Model-theoretic characterizations of arcs and simple closed curves," Proc. A. M. S. 104(1988), 898-904.

[4] -, "Co-elementary equivalence for compact Hausdorff spaces and compact abelian groups," J. Pure and Applied Algebra 68 (1990), 11-26.

[5] — , "Taxonomies of model-theoretically defined topological properties," J. Symbolic Logic 55(1990), 589-603.

[6] —— "Co-elementary equivalence, co-elementary maps, and generalized arcs," Proc. A. M. S. 125 (1997), 3715-3720.

[7] _ "On the topological model theory of normal disjunctive lattices," (submitted).

[8] — "A hierarchy of maps between compacta," J. Symbolic Logic (to appear).

[9] C. C. Chang and H. J. Keisler, "Model Theory (Third Edition)," North Holland, Amsterdam, 1990.

[10] D. Dacunha-Castelle and J. Krivine, "Applications des ultraproduits à l'étude des espaces et des algèbres de Banach," Studia Math. 41(1972), 315-334.

[11] P. Eklof, "Ultraproducts for algebraists," in "Handbook of Mathematical Logic," North Holland, Amsterdam, 1977, pp. 105-137.

[12] R. Engelking, "Outline of General Topology," North Holland, Amsterdam, 1968.

[13] R. Gurevič, "On ultracoproducts of compact Hausdorff spaces," J. Symbolic Logic 53(1988), 294-300.

[14] S. Heinrich and C. W. Henson, "Model theory of Banach spaces, II: isomorphic equivalence," Math. Nachrichten 125(1986), 301-317.

[15] S. Heinrich, C. W. Henson, and L. C. Moore, Jr., "A note on elementary equivalence of $C(K)$ spaces," J. Symbolic Logic 52(1987), 368-373.

[16] C. W. Henson, "Nonstandard hulls of Banach spaces," Israel J. Math. 25(1976), 108-144.

[17] C. W. Henson, "Nonstandard analysis and the theory of Banach spaces," Springer Lecture Notes in Math., No. 983 (1983), 27-112.

[18] C. W. Henson and J. Iovino, "Banach Space Model Theory, I," (lecture notes monograph, in preparation).

[19] W. Hodges, "Model Theory," Cambridge University Press, Cambridge, 1993.

[20] K. Kuratowski, "Topology, vol. II," Academic Press, New York, 1968.

[21] A. Macintyre, "Model completeness," in "Handbook of Mathematical Logic," North Holland, Amsterdam, 1977, pp. 139-180.

[22] S. B. Nadler, Jr., "Continuum Theory, An Introduction," Marcel Dekker, New York, 1992.

[23] W. V. O. Quine, "Word and Object," The M. I. T. Press, Cambridge, MA, 1960.

[24] J. Rosický, "Categories of models," Seminarberichte Mathematik Informatik Fernuniversität 19(1984), 337-413.

[25] S. Shelah, "Every two elementarily equivalent models have isomorphic ultrapowers," Israel $J$. Math. 10(1971), 224-233.

[26] G. F. Simmons, "Introduction to Topology and Modern Analysis," McGraw-Hill, New York, 1963.

[27] H. Wallman, "Lattices and topological spaces," Ann. Math.(2) 39(1938), 112-126.

[28] S. Willard, "General Topology," Addison-Wesley, Reading, MA, 1970. 